\title{
A Novel Approach on Color Image Refocusing and Defocusing
}

\author{
Atrikesh Pandey \\ Department of Computer \\ Science \\ SCERT Barabanki
}

\author{
Deepak Pant \\ Department of Computer \\ Science \\ GNITM Barabanki
}

\author{
Kapil Kumar Gupta \\ Department of computer \\ Science \\ AITM Lucknow
}

\begin{abstract}
In this study we present a novel approach on a color image refocusing and defocusing. In detail, the proposed method can accomplish the tasks of color image refocusing and defocusing. Our proposed method is thus a process of recovering the original color image from the degraded color image observations.

This approach has the advantage of canny edge detection process for preserving the sharp Edges and lines of color images for restoring the blurred color image. Blind deconvolution algorithm is used to blurred images where image recovery process has been performed with little or no prior knowledge of the degrading point spread function.
\end{abstract}

\section{General Terms}

Image processing, Color image focusing.

\section{Keywords}

Defocusing, Partial Occlusion, Refocusing, Deconvolution, Noise reduction

\section{INTRODUCTION}

Color image refocusing and defocusing process such as color image tend to get blurry, the blurred impression of the color images is due to the fact that image pixels are averaged with their neighbors. Blurred images don't have sharp boundaries. In color images, noise detection and removal is an important process as the images are corrupted by those noises because of transmission and acquisition. The main aim of the noise removal is to suppress the noise when preserving the edge information. However, the color images are likely to be corrupted by noise due to bad acquisition, transmission or recording. Such degradation negatively influences the performance of many image processing techniques and a preprocessing module to filter the images is often required, so in this way we often required the image refocusing and defocusing techniques. An image is a rectangular grid of pixels. It has a definite height and a definite width counted in pixels. Each pixel is square and has a fixed size on a given display[1].

However different computer monitors may use different sized pixels. Each pixel has a color. The color is a 32-bit integer. The first eight bits determine the redness of the pixel, the next eight bits the greenness, the next eight bits the blueness, and the remaining eight bits the transparency of the pixel. The other is defocusing, which is to blur an image and to create defocus effects. In some photography such as portrait, shallow depth of field is preferred so as to highlight the foreground subject with a defocused blurry background. However, due to the limitations of the lens and sensors, some cameras such as point-and-shoot cameras cannot produce enough defocus effects.
A different approach to refocusing is to measure the light field associated with an image. In this case, the measured rays can be combined to simulate new depth of field settings without explicitly computing depth. Levoy and Hanrahan [1996] compute a light field from a large number of images (between 256 and 4096) and use it to simulate synthetic camera apertures. This idea was further extended in [Isaksen et al. 2000][33] and [Levoy et al. 2004][34]. The deduction of this approach is that it either requires the sequential capture of a large number of images. In this paper, we have also developed a refocusing algorithm which considers partial occlusions at object boundaries. In particular, our algorithm defocuses image points by respecting visibility changes for different points on a large aperture lens, and by more accurately mixing foreground and background pixels in the defocus computation. The algorithm is used with the computed depth map to refocus either the original color image, or an image taken immediately before or after under different lighting.

The rest of the paper is structured as follows-In section 2, we review of the previous work. Section 3, gives a brief overview of our approach and problem formulation.

\section{RELATED WORK}

We review the relevant prior work on color image refocusing and prior work on blurriness of the color image.There is some causes of blurring a color image:

- Scattered light distortion in confocal microscopy.

- Movement during the image captures process.

The performance of the Canny edge algorithm depends heavily on the adjustable parameters, $\sigma(1.4)$, which is the standard deviation for the Gaussian filter, and the threshold values, 'T1' and 'T2'. $\sigma$ also controls the size of the Gaussian filter. The bigger the value for $\sigma$, the larger the size of the Gaussian filter becomes. This implies more blurring, necessary for noisy images, as well as detecting larger edges. The standard median filter (MF) [10] has been prevalently used in for noise reduction of image preprocessing. However, there are two inherent limitations of the MF. The first is high computation load. The second is that it removes the thin lines and small objects of interest and blurs the details even at low noise densities, while the size of the filter window increasing [11].

The defocusing there is handled with the aid of the lens blur tool in Photoshop. In this paper, a simple and wellparameterized multipoint scheme is adopted to measure the edge blurriness. In addition to defocusing, we also address the more challenging refocusing problem with a blind deconvolution framework. The edge information is exploited 
not only in focus detection but also in image refocusing in this paper.The method proposed by Bando and Nishita [8] can tackle the single-image refocusing task, but it requires a lot of user intervention to determine the blur kernel from a number of predefined candidates. While in this paper, the optimal blur kernel as well as the target refocused image is obtained automatically using a well-regularized blind deconvolution method.

A priori blur identification methods are the class of methods that perform the blind deconvolution by identifying the PSF prior to the restoration. Typically these methods assume the PSF to be of a known parametric form. The associated parameters are unknown and to be determined before restoration. This method is relatively simple but have some major drawbacks like they require the knowledge of the form of PSF, some PSFs simply don't have frequency zeros. Finally additive noise can mask the frequency domain nulls and thus degrade the performance [14].

Edge detection is the process of identifying and locating sharp discontinuities in an image. The discontinuities are abrupt changes in pixel intensity which characterizes boundaries of objects in a scene[36]. Classical methods of edge detection involve convolving the image with an operator (a 2-D filter), which is constructed to be sensitive to large gradients in the image while returning values of zero in uniform regions. There is an extremely large number of edge detection operators available, each designed to be sensitive to certain types of edges.

Image deblurring is an inverse problem whose aspire is to recover an image which has suffered from linear degradation. The blurring degradation can be space invariant or space in variant. Inverse problems are often ill-posed [8]. Existing blind deconvolution methods are a priori blur identification method, zero sheet separation method, ARMA parameter estimation methods and nonparametric methods [2].

\section{OUR APPROACH}

The canny edge detector first smoothes the color image to eliminate noise. It then finds the color image gradient to highlight regions. The algorithm then tracks along these regions and suppresses any pixel that is not at the maximum (non-maximum suppression). The gradient array is now further reduced by hysteresis. Hysteresis is used to track along the remaining pixels that have not been suppressed. Hysteresis uses two thresholds and if the magnitude is below the first threshold, it is set to zero (made a nonedge). If the magnitude is above the high threshold, it is made an edge. And if the magnitude is between the 2 thresholds, then it is set to zero unless there is a path from this pixel to a pixel with a gradient above $\mathrm{T} 2$.

As, shown in the fig. 1, the rays starting from the scene point $\mathrm{p}_{1}$ on the focal plane can converges to a point on the color image plane. However, when the scene point moves away $\left(\mathrm{p}_{2}\right)$ from the focal plane, the rays will give rise to a blur circle on the image plane, and the color image is regarded as defocused. When the point $\left(\mathrm{p}_{3}\right)$ moves farther, a blurrier defocused image is produced. Such blurring process is often modeled as the convolution of the focused image $F$ with PSF, i.e.

$$
\mathrm{I}=\mathrm{F}^{*} \mathrm{~h}+\mathrm{n}
$$

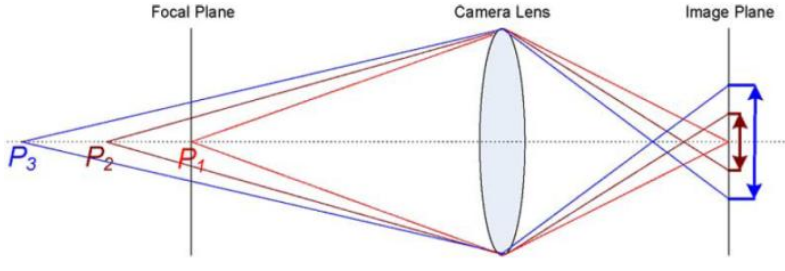

Fig 1: Geometry of the imagining model. $P_{1}, P_{2}$ and $P_{3}$ represent the scene points at the different depth.

Where, I denote the defocused image and $\mathrm{n}$ is the noise term. Due to the diffraction and aberration of the camera lens, the PSF is approximated normally by a 2-D Gaussian filter [12] [13], The spread parameter , which is related to the distance of the object to the focal plane, determines the blurriness of the captured color image. In this paper, the above given formula is used to model the deblurringss process.

Canny edge detection method finds edges by looking for local maxima of the gradient of $f(x, y)$. The gradient is calculated using the derivative of a Gaussian Filter [Gaussian filter is used to blur an image using Gaussian function. It requires two parameters such as mean and variance. It is weighted blurring. Gaussian function is of the following form where $\sigma$ is variance and $\mathrm{x}$ and $\mathrm{y}$ are the distance from the origin in the horizontal axis and vertical axis respectively. Gaussian Filter has an efficient implementation of that allows it to create a very blurry blur image in a relatively short time.]. The method uses two thresholds to detect strong and weak edges, and includes the weak edges in the output only if they are connected to strong edges. Therefore, this method is more likely to detect true weak edges.

Steps by step canny method approach -

- The color image is smoothed using Gaussian Filter with a specified standard deviation, $\sigma$, to reduce noise.

- The local gradient, $\mathrm{g}(\mathrm{x}, \mathrm{y})$ and edge direction are computed at each point.

- The edge point determined give rise to ridges in the gradient magnitude image. This ridge pixels are then thresholds, T1\& $\mathrm{T} 2$, with $\mathrm{T} 1<\mathrm{T} 2$. Ridge pixels with values greater than $\mathrm{T} 2$ are said to be 'strong' edge pixels. Ridge pixels with values between T1 \& T2 are said to be 'weak' edge pixels.

\subsection{Deblurring Color Image Using The Blind Deconvolution Algorithm}

Compared with defocusing, refocusing is more challenging. Image deblurring can be done by the different blurring technique like Gaussian blur.

For blurring an color image some parameters are required blur length, PSF(point spread function),blur angle. Blur length is the number of pixels by which the image is degraded. PSF is when the intensity of the observed point image is spread over several pixels. Blur Angle is that angle at which the image is degraded.

The Blind Deconvolution Algorithm can be used effectively restoring a color image when no information about the distortion (blurring and noise) is available. In this, restores the color image and the point-spread function (PSF) simultaneously. 
Algorithm for Deblurring -

Input:

Load an input color image "f"

Initialize blur length "bl"

Initialize blur angle "theta $\theta$

Assign the type of noise "n

PSF (Point Spread Function), "h"

$\mathrm{h}=$ create(f, bl, theta $\theta) \%$ Creation of PSF

Blurred image $(\mathrm{g})=\mathrm{f} * \mathrm{~h}+\mathrm{n}$

Initialize number of iterations " $i$ "

Weight of an color image "w" \%pixels considered for restoration

$\mathrm{a}=0$ (default) \%Array corresponding to additive noise

Procedure $-\mathrm{i}$

If PSF is not known then, Guess initial value of PSF

Else

Specify the PSF of degraded color image

Restored Image "f"= Deconvolution (g, h, i, w, a)

End Procedure - i

The PSF is a delta kernel, and the latent color image is the same with the observed blurry one.In addition, proper user intervention is often required at the initialization stage, and poor initialization may result in undesired local convergence. Although some efforts were made to seek the PSFs from the edges, the edge sharpness cue is not utilized adequately.

In this paper, we present a refocusing method that takes full advantage of the canny edge sharpness cue. First, it is utilized for PSF estimation. Then, edge sharpness prior is developed to constrain the PSF not to blur the edges and enforce the refocusing color image to agree with the precalculated sharpened color image in the vicinity of edges.

\subsection{Architecture Of Deblurring Algorithm}

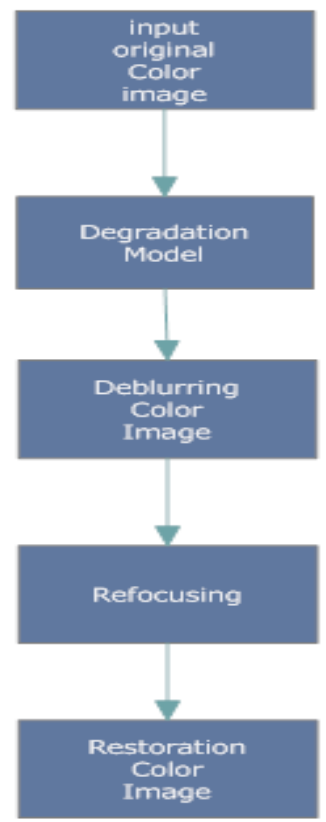

Fig 2: Overall Architecture Of Deblurring Algorithm

The following fig. 2.represents, the overall architecture of this paper. The original color image is degraded using degradation model to produce the blurred color image. The blurred image should be an input to the Deblurring model [A blurred or degraded color image can be approximately describe by this equation: $\mathbf{g}=\mathbf{h} \mathbf{f}+\mathbf{n}$ where, "g" is blurred color image, " $\mathrm{h}$ " is point spread function (PSF), " $\mathrm{f}$ " is original color image and " $n$ " is additive noise in color image]. In this paper, we are going to use Blind Deconvolution Algorithm for deblurring a color image. The result of this algorithm produces the deblurring image which can be compared with our original image.

\subsection{Refocusing}

The color image may be refocused with different focal panel and aperture settings, by convolving each pixel. Realistic depth of field rendering is achieved by taking into account partial occlusion at object boundaries.

In particular, let us say we wish to refocus a color image with three types of regions:

- a region $F$ (foreground) with depth $d_{F}$,

- a region $B$ (background) with depth $d_{B}$ and,

- a region $C$ (boundary) with a depth that smoothly changes from $d_{F}$ to $d_{B}$.

Partial occlusions: Consider the scenario illustrated in Fig.3; we want to compute the irradiance of an color image pixel $p$ which receives light from a lens with a large aperture, focused behind the scene. Two objects A and B are in the field of view of $\mathrm{p}$, where Ais locatedin front of B. The total light energy received by $\mathrm{p}$ is the sum of the contributions of all the light rays from the lens. The contributions of these rays can be determined by tracing the rays from the lens to points on the surfaces of A and B. In our case, however, we are given a single, narrow-aperture view ofthe scene and the corresponding depth map. There could be regions of the objects $\mathrm{A}$ and $\mathrm{B}$ that contribute to the irradiance of pixel pin the refocused color image, that are not captured in the acquired color image.This is illustrated in Fig.3 where the acquired color image is assumed tobe an orthographic view of the scene (dotted horizontal lines). In this case, although we need the radiances of the points on object $\mathrm{B}$ that lie between $\mathrm{b} 2$ and $\mathrm{b} 3$, they are not included in the acquired color image. We recreate such missing regions by detecting discontinuitiesin our depth map and extending the occluded surface using texture synthesis.

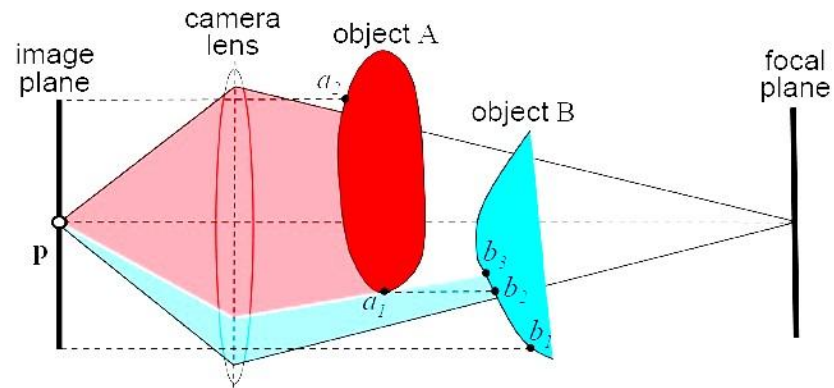

Fig 3: The problem of partially occlusions associated with rendering a refocused image with a wide aperture given a single all focused image and a depth map.

First we compute $\mathbf{R}_{C 2 F}$ where we have assigned a depth $d_{F}$ to all the points in $C$. Thesecond refocused image, $\mathbf{R}_{C 2 \mathrm{~B}}$, is computed by assigning a depth $d_{B}$ to the pixels in $C$. The final refocused image is computed as

$\mathbf{R}=\mathbf{R}_{C 2 F} * \mathbf{A}+\mathbf{R}_{C 2 B} *(\mathbf{1}-\mathbf{A})$

Where, $\mathbf{1}$ is a matrix of ones of the same size as $\mathbf{A}$, and * denotes element-wise multiplication. 


\section{EXPERIMENTAL RESULTS}

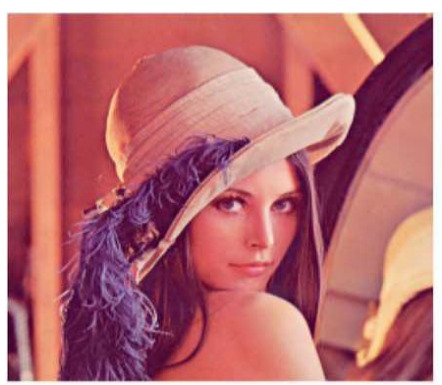

Fig 4: Original Color Image

The above color images represent the result of degradation model using Gaussian blur. First image represents the original image and its edge can be estimated by Canny Edgedetection method.

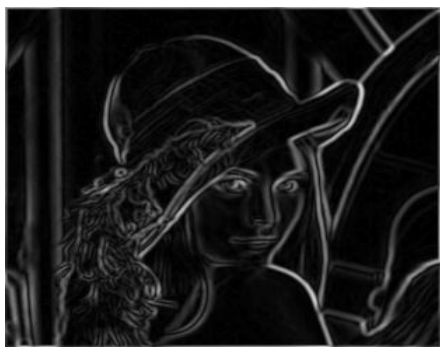

Fig 5: Edges of Original Color Image

The edge detection can be applicable to Gray Image. Therefore, the original RGB image can be converted to gray image. After that Canny Edge Detection is applied for getting the Edges of the original image.

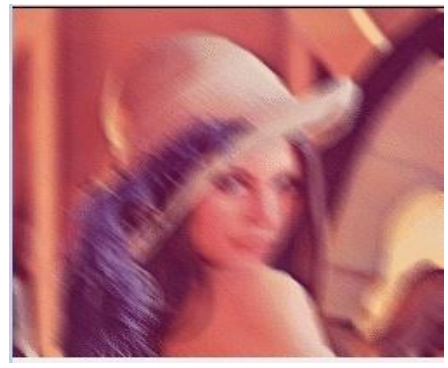

Fig 6: Blurred Color Image

The original color image can be blurred using Gaussian low pass filter by specifying the blur parameters. The following image is depicted as blurred color image.

Image after applying the proposed algorithm will be as follows.

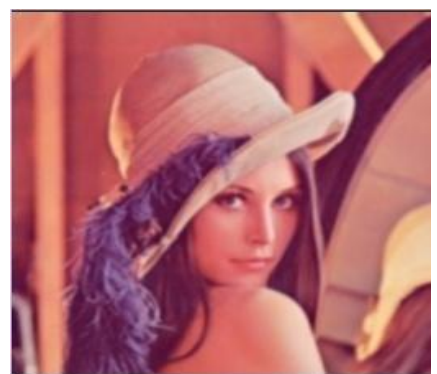

Fig 7: Restored Color Image

\section{CONCLUSIONS}

This paper, we have presented a system to handle the tasks of color image refocusing and defocusing. First, by means of a parametric edge model, we propose an efficient and effective canny edge detection method. Second, the challenging refocusing problem is tackled in partial occlusion. A wide variety of color images has been tested to validate the proposed algorithm. The main limitations of the method arise from the sparsity of the depth estimation and errors in the initial segmentation of the color image. Despite these limitations, the method is applicable to a wide variety of scenes as evidence by our experimental results.

\section{ACKNOWLEDGMENTS}

Our thanks to the experts who have contributed towards development of the template. This work is supported by resources from Prof. Dr. Devendra Agarwal Director GITM Lucknow and Ms. Namrata Dhanda Head of department of computer science and engineering and Information Technology, GITM, Lucknow. They would also thank the anonymous reviewers for their significant and constructive critiques and suggestions, which substantially improved the quality of this paper.

\section{REFERENCES}

[1] Wei Zhang, Wai-Kuen Cham,"Single image refocusing and defocusing" IEEE Transaction. on image processing, vol.21, NO. 2, FEBRUARY 2012.

[2] P. Campisi and K. Egiazarian, "Blind Image Deconvolution: Theory and Applications." Boca Raton, FL: CRC, 2007.

[3] T. Y. Sun, S. J. Ciou, C. C. Liu, C. L. Huo, "Out and -ofFocus Blur Estimation for Blind Image Deconvolution: Using Particle Swarm Optimization," in Proc. 2009 International Conference on Systems, Man and Cybernetics, pp. 1627-1632, San Antonio, Texas, USA, Oct. 14-18,2009.

[4] S.Ramya and T.Mercyl, "Restoration of blurred images using Blind Deconvolution algorithm" in Proc.: Emerging Trends in Electrical and Computer Technology(ICETECT),2011 International Conference, pp. 496499, Tamilnadu, India, March 23-24,2011

[5] D. Kundur and D. Hatzinakos, "A novel blind deconvolution scheme for image restoration using recursive filtering," IEEE Trans. on Signal Processing, vol. 46, no. 2, pp. 375-390, 1998.

[6] C. Vural and W. A. Sethares, "Blind image deconvolution via dispersion minimization," Digital Signal Processing, vol. 16, pp. 137-148, 2006.

[7] F. Moreno-Noguer, P. N. Belhumeur, and S. K. Nayar, "Active refocusing of images and videos," in Proc. SIGGRAPH, 2007.

[8] Y. Bando and T. Nishita, "Towards digital refocusing from a single photograph," in Proc. Pacific Graph., 2007, pp. 363-372.

[9] Almeida, M.S.C.; Almeida, L.B.;, "Blind and SemiBlind Deblurring of Natural Images," IEEE Trans. on Image Processing, vol.19, no.1, pp.36- 52, Jan. 2010.

[10] AI. Bovik, Handbook of Image \& Video Processing., seconded., Elsevier Academic Press, 2005.

[11] Kapil Kumar Gupta, M. Rizwen Beg, Jitendra Kumar Niranjan, "A novel approach to fast image filtering algorithm of infrared images based on intro sort algorithm" IJCSI 8-6-1Nov. 2011.

[12] H. Y. Lin and C. H. Chang, "Depth from motion and defocus blur,”Opt. Eng., vol. 45, no. 12, p. 127 201, Dec. 2006. 
[13] P. Favaro and S. Soatto, "A geometric approach to shape from defocus,"IEEE Trans. Pattern Anal. Mach. Intell., vol. 27, no. 3, pp. 406-417, Mar. 2005.

[14] Rupali Patil, Sageeta Kulkarni,;"'Blurred image restoration using canny edge detection and blind deconvolution algorithm" IJCTEE

[15] Francesc Moreno-Noguer, Peter N. Belhumeur, Shree K. Nayar, ; "Active refocusing of image and videos"

[16] G. L. Fan and W. K. Cham, "Model-based edge reconstruction for low bit-rate wavelet-compressed images," IEEE Trans. Circuits Syst. Video Technol., vol. 10, no. 1, pp. 120-132, Feb. 2000.

[17] J. Canny, "A computational approach to edge detection," IEEE Trans. Pattern Anal. Mach. Intell., vol. PAMI-8, no. 6, pp. 679-698, Nov. 1986.

[18] E. Eisemann and F. Durand, "Flash photography enhancement via intrinsic relighting," in Proc. SIGGRAPH, 2004, pp. 673-678.

[19] G. Petschnigg, R. Szeliski, M. Agrawala, M. Cohen, H. Hoppe, and K. Toyama, "Digital photography with flash and no-flash image pairs," in Proc. SIGGRAPH, 2004, pp. 664-672.

[20] A. Levin, D. Lischinski, and Y. Weiss, "Colorization using optimization," in Proc. SIGGRAPH, 2004, pp. 689-694.

[21] T. F. Chan and C. K. Wong, "Total variation blind deconvolution," IEEE Trans. Image Process., vol. 7, no. 3, pp. 370-375, Mar. 1998.

[22] N. Joshi, R. Szeliski, and D. J. Kriegman, "PSF estimation using sharp edge prediction," in Proc. IEEE Conf. CVPR, 2008, pp. 1-8.

[23] R. Fergus, B. Singh, A. Hertzmann, S. T. Roweis, and W. T. Freeman, "Removing camera shake from a single photograph," in Proc. SIGGRAPH, 2006, pp. 787-794.

[24] N. Joshi, C. L. Zitnick, R. Szeliski, and D. J. Kriegman, "Image deblurring and denoising using color priors," inProc. IEEE Conf. CVPR, 2009, pp. 1550-1557.
[25] Q. Shan, J. Y. Jia, and A. Agarwala, "High-quality motion deblurring from a single image," in Proc. SIGGRAPH, 2008.

[26] J. Y. Jia, "Single image motion deblurring using transparency," in Proc. IEEE Conf. CVPR, 2007, pp. 18.

[27] A. Levin, Y. Weiss, F. Durand, and W. T. Freeman, "Understanding and evaluating blind deconvolution algorithms," in Proc. IEEE Conf. CVPR, 2009, pp. 1964- 1971.

[28] Y.Wang, J. Yang, W. Yin, and Y. Zhang, "A new alternating minimization algorithm for total variation image reconstruction," Rice Univ. CAAM, Houston, TX, Tech. Rep., 2007.

[29] E. van den Berg and M. P. Friedlander, "Probing the Pareto frontier for basis pursuit solutions," SIAM J. Sci. Comput., vol. 31, no. 2, pp. 890-912, 2008.

[30] E. van den Berg and M. P. Friedlander, "SPGL1: A solver for largescale sparse reconstruction," 2007 [Online]. Available: http://www.cs.ubc.ca/labs/scl/spgl1

[31] Z. Wang, A. C. Bovik, H. R. Sheikh, and E. P. Simoncelli, "Image quality assessment: From error visibility to structural similarity," IEEE Trans. Image Process., vol. 13, no. 4, pp. 600-612, Apr. 2004.[32] Q. Hou, K. Zhou, and B. Guo, "BSGP: Bulk- synchronous GPU programming," in Proc. Siggraph, 2008, pp. 1-12.

[33] Isaksen, A., Mcmillan, L., and Gortler, S. J. 2000. Dynamically reparameterized light fields. In Proc. Siggraph, 297.306.

[34] Levoy, M., and Hanrahan, P. 1996. Light field rendering. In Proc. Siggraph, 31.42 .

[35] Levoy, M., Chen, B., Vaish, V., Horowitz, M., dowall, I., and Bolas, M. 2004. Synthetic aperture confocal imaging. In Proc. Siggraph, 825.834.

[36] R.Maini, H.Aggarwal. Study and comparison of various image edge detection techniques, International Journal of Image processing (IJIP), volume (3), issue (1) 2009. 